\title{
Pequeños exploradores de la ciencia: una propuesta pedagogica para el desarrollo del pensamiento científico en niños de nivel preescolar*
}

\section{Little explorers of science: A pedagogical proposal for development of scientific thinking in preschool children}

\author{
Íngrid Johanna Rojas Mercado ${ }^{1 \mathbb{D}}$, Elda Cerchiaro Ceballos ${ }^{\mathbb{D}}$
}

\begin{abstract}
Para citar este artículo: Rojas, Í., Cerchiaro, E. (2020). Pequeños exploradores de la ciencia: una propuesta pedagógica para el desarrollo del pensamiento científico en niños de nivel preescolar. Infancias Imágenes, 19(2), 80-95
\end{abstract}

\section{Resumen}

La presente investigación se centra en el desarrollo del pensamiento científico en niños de nivel preescolar
Recibido: 16-04-2019 - Aceptado: 25-05-2020

\begin{abstract}
This research focuses on the development of scientific thinking in preschool children, through a pedagogical proposal that promotes the development of cognitive skills and scientific reasoning. A mixed methodology was used, with a quasi-experimental design with a single group, of the pretest-posttest type, in which the Merrill-Palmer development scale (MP-R) of Roid and Sampers was used, before and after the implementation of the proposal. The analysis of the information compares the results using the Wilcoxon signed rank statistical test to establish the relationship between the pedagogical proposal and the change in cognitive abilities. The results indicate changes in the profiles of cognitive abilities associated with the participation of children in the pedagogical proposal. It is concluded that the teaching of science from preschool, by strengthening cognitive skills, favors the development of scientific thinking.
\end{abstract}

Keywords: science, thinking, skills, pedagogical research, childhood.

Palabras clave: ciencia, pensamiento, habilidades, investigación pedagógica, infancia.

\footnotetext{
* En este artículo se presentan los resultados de la investigación "Una propuesta pedagógica para la enseñanza de las ciencias en el nivel de preescolar", desarrollada en la institución educativa departamental del Magdalena Cerro Blanco, en el marco del convenio de la Universidad del Magdalena y la Secretaría Departamental del Magdalena, en el periodo de enero de 2017 a abril de 2019, como opción de grado para optar al título de magister en Educación en la Universidad del Magdalena por parte de una de las autoras.

1 Magister en Educación, Universidad del Magdalena. Licenciada en educación preescolar, Universidad del Magdalena. Correo electrónico: ingrojasmer1984@gmail.com. Orcid: https://orcid.org/0000-0003-4631-5297.

2 Doctorado en Psicología, Universidad Del Valle. Magister, Universidad Externado de Colombia. Psicóloga, Universidad Metropolitana. Correo electrónico: elda.cerchiaro@gmail.com. Orcid. https://orcid.org/0000-0002-6440-4659.
} 


\section{Introducción}

Pensar es una actividad propiamente humana (Rodríguez, 2011). Partiendo de esta premisa, se puede afirmar que el ser humano, a diferencia de los animales, piensa cuando realiza actividades que involucran el aprendizaje, la toma de decisiones y la solución de problemas. Las anteriores son actividades cognitivas que se constituyen en un objetivo de primer orden en la educación de la primera infancia para el desarrollo del pensamiento científico. De allí que se considere fundamental su promoción en los primeros años de vida, por el impacto que produciría en la vida escolar de niños y jóvenes.

En ese contexto, la dificultad de la enseñanza de las ciencias se convierte hoy día en un reto para la educación y tratarla desde los primeros años de escolaridad es una prioridad. Históricamente, el educar en ciencias se ha apoyado en la idea tradicional de una enseñanza basada en la exposición y trasmisión de contenidos, y desde el preescolar se ha constituido en un concepto vacío o desconocido, pues poco se trabaja o se fomenta en nuestras escuelas. Parece olvidarse que la ciencia no solamente facilita las condiciones de vida, sino que incluye aspectos como experimentar, observar, comprobar, estudiar fenómenos, relacionarse con lo científico, entre otras actividades, que favorecen el logro de aprendizajes desde el hacer, crear y conocer (Ausubel et al., 1983).

A su vez, resulta evidente encontrar en las escuelas rurales la insuficiente y limitada posibilidad de crear espacios en los que niños y niñas tengan la oportunidad de investigar y crear. Ello no permite dar respuesta a su necesidad de indagar, descubrir y pensar científicamente, pues se entiende que "el desarrollo de las ideas de los niños pequeños se construye a partir de la oportunidad de experiencias que se les brinden" (Harlen, 1998, p. 68). Pues, se parte de reconocer, como señala Gopnik (citada en Winerman, 2017) que los niños son aprendices inventivos que adquieren el conocimiento que necesitan a través del juego creativo y la observación del mundo que los rodea.

La presente investigación se centra en la manera como actualmente se aborda la enseñanza de las ciencias en el nivel de preescolar, caracterizada por: las limitadas estrategias escolares para enseñar la ciencia, la ausencia de espacios donde los niños puedan crear e investigar, el débil aprovechamiento de su contexto, la poca credibilidad en las capacidades científicas de los niños, el desconocimiento del verdadero sentido de la educación científica como desarrollo del pensamiento científico desde la edad preescolar. Todo lo anterior pasando por alto la necesidad de trascender en el aporte que se les ofrece para que puedan lograr aprendizajes significativos en su proceso de formación.

Ante esta situación surge la siguiente pregunta de investigación: ¿cómo desarrollar el pensamiento científico en niños de nivel preescolar a partir de una propuesta pedagógica para la enseñanza de las ciencias en una institución educativa departamental? Se pretende estructurar un modelo que oriente la enseñanza de las ciencias desde el preescolar, como una experiencia pedagógica válida y significativa que puede ser aprovechada por otros docentes. Al mismo tiempo, se busca promover en los niños capacidades que les permitan ser sujetos curiosos, reflexivos y críticos, con disposición para indagar, experimentar y descubrir por cuenta propia.

Gracias a los aportes de disciplinas como las neurociencias, psicología del desarrollo, neuroeducación, entre otras, hoy día se reconoce la importancia que las experiencias tempranas en la infancia tienen en el desarrollo cerebral, motor, cognitivo, socio afectivo, comunicativo y lingüístico de los niños y niñas (Bick, 2015; Cypel, 2013; Torrado et al., 2006). En ese contexto adquiere mayor importancia la educación preescolar, pues es cada vez más reconocida en todo el mundo (Yañez, 2013). La educación preescolar se constituye en un medio fundamental para el desarrollo humano de un país; por tanto, los esfuerzos que se hagan para promover el aprendizaje de las ciencias desde la primera infancia resultan una tarea inaplazable (De Zubiría, 2013).

En este mismo sentido, los aportes científicos sobre la promoción del pensamiento científico y habilidades cognitivas a partir de la enseñanza de las ciencias señalan la pertinencia de iniciar desde las primeras edades, con el fin de aprovechar la curiosidad, capacidad de asombro y el potencial que estos niños y niñas tienen para el aprendizaje, 
ofreciéndoles espacios formativos que permitan potenciar sus capacidades cognitivas (Ausubel et al., 1983; Piaget, 1969; Vigotsky, 1979). La presente investigación pretende avanzar en esta dirección a través de una propuesta pedagógica enfocada en promover el pensamiento científico en niños del nivel preescolar.

Se trata de un estudio mixto en el que se utilizó un diseño cuasiexperimental con un solo grupo, de tipo pretest-postest (Hernández et al., 2006), con la cual se evaluaron las habilidades cognitivas de los niños participantes a partir de la escala de desarroIlo Merrill-Palmer (MP-R) de Roid y Sampers (2004), antes y después de la aplicación de la propuesta pedagógica. La información que ofrece la escala sobre cada niño es complementada con datos cualitativos recogidos mediante diarios de campo que permiten reconstruir cada experiencia de las actividades pedagógicas desarrolladas con los niños. El análisis de toda esta información permitió identificar cuáles son las condiciones (individuales, peda-

82 gógicas, didácticas y contextuales) que favorecen el desarrollo del pensamiento científico en niños preescolares en la escuela que ha sido escenario de la investigación.

\section{Diseño y desarrollo metodológico}

El presente estudio se enmarca en el paradigma empírico-analítico, desde el cual se asume una postura pragmática que acoge la observación, medición y verificación del fenómeno a estudiar como herramientas que conducen a la caracterización de condiciones a partir de las cuales, en la realidad escolar estudiada, se puede promover el pensamiento científico en niños de nivel preescolar.

Se adopta una metodología de tipo mixto, en la que se realiza un proceso de recolección, análisis y vinculación de datos cuantitativos y cualitativos con el fin de dar respuesta a la pregunta problema planteada (Hernández et al., 2006). Esta metodología que, según Samper, (2013) se constituye como "el cruce de los enfoques", permite aplicar procesos cuantitativos y cualitativos a los datos recolectados, los análisis efectuados y las explicaciones y conclusiones alcanzadas, con el fin de lograr una perspectiva más precisa del fenómeno estudiado. El enfoque mixto permite amplitud, mayor profundidad, diversidad, riqueza interpretativa y sentido de entendimiento de la problemática planteada (Creswell et al., 2005; Teddlie y Tashakkori, 2003, citados en Hernández et al., 2006).

Desde esta perspectiva, en la presente investigación la información cuantitativa se obtuvo a partir de los resultados de la aplicación de una prueba que evaluó habilidades cognitivas en los niños participantes, pues dio los insumos estadísticos necesarios que conducen a conclusiones relevantes del estudio. Estos datos se complementan con la información cualitativa derivada del uso de la observación mediante diarios de campo y filmaciones, tanto como un medio fiable para registrar y reconstruir la experiencia de las actividades pedagógicas desarrolladas con los niños, en términos de las interacciones, verbalizaciones, expresiones emocionales, actitudes, motivación, etc., así como indicadores de su participación.

En esta investigación se utilizó un diseño cuasi experimental de un solo grupo, con mediciones pretest y postest, mediante una evaluación de habilidades cognitivas de los niños participantes en el estudio, antes y después de la implementación de una propuesta pedagógica para la enseñanza de las ciencias. Este diseño permite establecer los cambios que se producen en las habilidades cognitivas de los niños después del desarrollo de la propuesta pedagógica, los cuales se asocian con su participación en las actividades desarrolladas. Dados los alcances del estudio no se efectuó control de variables extrañas; sin embargo, se estableció como medida de control que el desarrollo de las actividades pedagógicas con los niños se hiciera únicamente en las clases de ciencias, de manera que los niños estuviesen expuestos a esta metodología de enseñanza nueva para ellos solamente en ese espacio curricular.

La propuesta pedagógica denominada "Pequeños exploradores de la ciencia" se desarrolló en tres meses durante los cuales se realizaron 16 actividades, con una intensidad de una hora aproximadamente por cada una. En ella participaron la totalidad de niños matriculados en el nivel de preescolar de la institución educativa. Se realizaron observaciones de cada actividad por medio de filmaciones y diarios de campo, los 
cuales permitieron reconstruir los momentos más importantes para los niños de las experiencias científicas.

En el presente trabajo, en lo fundamental, se estudian dos variables (tabla 1): 1) la propuesta denominada "Pequeños exploradores de la ciencia", para la enseñanza de ciencias en niños de nivel preescolar, como medio para potenciar su pensamiento científico; y 2) las habilidades cognitivas evaluadas a través de la escala cognitiva de las escalas de desarrollo Merrill-Palmer (MPR) (Roid y Sampers, 2004). Se exploró la relación entre estas dos variables, en tanto la propuesta pedagógica fue la variable independiente, es decir, como "causa supuesta de la variable dependiente" (Kerlinger y Lee, 2002, p. 42), esto es, del cambio en las puntuaciones de las habilidades cognitivas de los niños.

Tabla 1. Operacionalización de las variables

\begin{tabular}{|c|c|c|c|}
\hline Variable & Operacionalización & Indicador & Instrumento \\
\hline $\begin{array}{l}\text { Propuesta pedagógica } \\
\text { “Pequeños exploradores } \\
\text { de la ciencia" para } \\
\text { la enseñanza de las } \\
\text { ciencias en el nivel } \\
\text { preescolar. }\end{array}$ & $\begin{array}{l}\text { Es un conjunto de experimentos y estrategias lúdicas } \\
\text { que tienen como fundamento las orientaciones } \\
\text { conceptuales del Ministerio de Educación Nacional } \\
\text { sobre el desarrollo de las competencias científicas } \\
\text { en niños de nivel preescolar y la metodología de } \\
\text { Scientific Thinking: Step by Step (Pensamiento } \\
\text { científico: paso a paso) (Booth, 2017). Se compone } \\
\text { de } 16 \text { actividades que se estructuran sobre los } \\
\text { siguientes pasos: } \\
\text { Primer paso: observar. Mirar de cerca, notar desde } \\
\text { diferentes puntos de vista y esperar sin mucho } \\
\text { "hacer". } \\
\text { Segundo paso: comparar. Hacer comparaciones para } \\
\text { ir más allá de decir lo que notaron acerca de algo y } \\
\text { comenzar a expresar relaciones entre las cosas. } \\
\text { Tercer paso: ordenar y organizar. Agrupar cosas por } \\
\text { rasgos reconocibles. Los niños emparejan, agrupan y } \\
\text { organizan materiales de muchas maneras diferentes. } \\
\text { Cuarto paso: predecir. Cuestionamiento y } \\
\text { especulación; basado en el conocimiento previo } \\
\text { derivado de los tres primeros pasos. } \\
\text { Quinto paso: experimentar. Los niños prueban sus } \\
\text { predicciones y sus ideas. } \\
\text { Sexto paso: evaluar. Los niños comunican los } \\
\text { resultados de sus experimentos a otros, tomando } \\
\text { su experiencia concreta, verbalizándola y } \\
\text { representando la información. } \\
\text { Séptimo paso: aplicar. Aplicar los conocimientos } \\
\text { obtenidos del experimento a un campo más amplio } \\
\text { de experiencia. }\end{array}$ & $\begin{array}{l}\text { Interacciones, } \\
\text { verbalizaciones, } \\
\text { expresiones } \\
\text { emocionales, actitudes } \\
\text { y motivación de los } \\
\text { niños manifestados } \\
\text { durante su } \\
\text { participación en las } \\
\text { actividades. }\end{array}$ & $\begin{array}{l}\text { Diarios de campo y } \\
\text { filmaciones. }\end{array}$ \\
\hline Habilidades cognitivas & $\begin{array}{l}\text { Razonamiento deductivo e inductivo, formación } \\
\text { de categorías/emparejamiento, comprensión } \\
\text { de analogías y relaciones, comprensión de } \\
\text { conceptos (todo/parte; igual/diferente), manejo } \\
\text { de la información secuencial, reconocimiento } \\
\text { de patrones, utilización del conocimiento } \\
\text { contextual, rastreo/búsqueda visual, manejo de } \\
\text { las interferencias, memoria de trabajo y atención } \\
\text { selectiva y dividida. }\end{array}$ & $\begin{array}{l}\text { Puntuaciones obtenidas } \\
\text { en la escala cognitiva } \\
\text { antes y después } \\
\text { del desarrollo de la } \\
\text { propuesta pedagógica. }\end{array}$ & $\begin{array}{l}\text { Escalas de } \\
\text { desarrollo Merrill- } \\
\text { Palmer (MP-R) } \\
\text { (Roid y Sampers, } \\
\text { 2004). }\end{array}$ \\
\hline
\end{tabular}

Fuente: elaboración propia. 
La población hacia la cual se enfocó esta investigación estuvo constituida por la totalidad de niñas y niños del nivel preescolar de una institución educativa departamental de la zona bananera en el departamento del Magdalena. Por su parte, la muestra se conformó inicialmente por 18 escolares (13 niñas y cinco niños) matriculados en el nivel preescolar de la sede Concepción, con edades comprendidas entre tres y seis años (edad promedio 4,9). Cuatro de estos niños abandonaron la escuela por factores no asociados a este proceso, tales como poco compromiso y esfuerzo por parte de los padres de familia y escasa motivación del alumno para asistir a clases. A estos se suman otros factores sociales como el traslado de domicilio por las condiciones de vida o por trabajo.

La selección de los niños y niñas se hizo atendiendo a los siguientes criterios:

- Estudiantes matriculados en el nivel de preescolar, jornada de la mañana en una institución educativa departamental de la zona bananera del departamento del Magdalena.

- Con edades entre tres y seis años.

- Participación autorizada por el padre, madre o acudiente responsable del niño o niña.

- Asistencia regular y puntual a la escuela.

Cabe aclarar que el padre, madre o adulto responsable de los niños y niñas, de manera libre y voluntaria firmó un acta de consentimiento informado en la cual se autorizó la participación de sus acudidos para ser filmados, fotografiados y entrevistados durante el desarrollo de la investigación.

Como técnica para la recolección de datos se utilizó una evaluación tipo test en dos momentos: una previa a la intervención (pretest) consistente en la implementación de una propuesta pedagógica y otra posterior (postest) para registrar cambios en la variable habilidades cognitivas que pudieran asociarse con la intervención realizada. Además, se usó la observación para registrar el desarrollo de las actividades contempladas en la propuesta pedagógica, de acuerdo con las exigencias que supone la complejidad de cada experiencia.

Las escalas de desarrollo Merrill-Palmer (MPR) (Roid y Sampers, 2004) fueron utilizadas como instrumento cuya finalidad es la evaluación global del desarrollo infantil, a través de la exploración de cinco grandes áreas del desarrollo del niño: cognición, motricidad, lenguaje y comunicación, conducta adaptativa y desarrollo socioemocional. Esta batería de pruebas es de aplicación individual, para niños de 0:1 a 6:6 años, en este caso se aplicó a niños entre tres y seis años, que plantea actividades y tareas muy participativas y atractivas, con escasas demandas de lenguaje expresivo y la utilización de juguetes, láminas con coloridas ilustraciones o la imitación de ciertos movimientos realizados por parte del examinador, lo que permite captar y mantener la atención incluso de los niños más pequeños. Su aplicación se realizó en las instalaciones de la institución educativa durante la jornada escolar, para lo cual se requirió de 30 a 40 minutos por sesión.

Las escalas MP-R permiten hacer interpretaciones tanto cuantitativas como cualitativas a partir de los resultados de las estimaciones de capacidad en puntuaciones típicas o en las puntuaciones de desarrollo, basadas en el modelo de Rasch que establece la probabilidad de respuesta del niño ante un ítem en términos de la diferencia entre la medida de rango del niño y la medida del ítem utilizado. Se utilizan las MP-R en su adaptación española (Arribas y Pereña, 2011) cuya tipificación se hizo con una muestra representativa de la población infantil española de 0:1 a 6:6 años compuesta por 968 niños procedentes de diversas regiones. Los parámetros construidos a partir de esta muestra de niños españoles ofrecen puntuaciones típicas, percentiles, puntuaciones de desarrollo y edades equivalentes (Roid y Sampers, 2004). Por las bondades que ofrece, las MP-R son utilizadas en la detección de trastornos del desarrollo (Alcantud et al., 2015; Alonso, 2014; Rico, 2013). En el contexto latinoamericano estas escalas han sido utilizadas para la evaluación del desarrollo con población hispanohablante en países como Ecuador y Colombia (Jácome-Vera, 2016; Monsalve et al., 2015). Para validar el uso del instrumento con la población a la que pertenecen los participantes de esta investigación, se realizó un estudio piloto con una muestra de 28 niños del mismo rango de edad de la muestra, pertenecientes a otra escuela pública ubicada en el mismo sector, la cual arrojó valores 
satisfactorios, con un alfa de Cronbach 0,852 con todas las variables de la prueba.

De acuerdo con el objeto de la presente investigación, se aplica únicamente la escala cognitiva (C) para evaluar el desarrollo de las habilidades cognitivas del niño, las cuales incluyen específicamente la cognición, la motricidad fina y el lenguaje receptivo; y, a su vez, arroja las siguientes puntuaciones: índice global (IG), cognición (C), motricidad fina (MF) y lenguaje receptivo (LR). También incluye tres escalas complementarias: memoria $(M)$, velocidad de procesamiento $(\mathrm{V})$ y coordinación visomotora (VM). Las escalas de la batería cognitiva tienen una fiabilidad adecuada superior a 0,85 en casi todas las escalas, y se pueden expresar en puntuaciones con una media de 100 y una desviación típica de 15 (Roid y Sampers, 2004, p. 21). Los resultados obtenidos serán presentados más adelante.Como se ha señalado, después de la evaluación pretest se implementó la propuesta pedagógica "Pequeños exploradores de la ciencia" la cual se basa en el diagnóstico previo realizado a los niños durante las primeras semanas de ingreso a la escuela. Una semana después de dar por finalizadas las actividades de la propuesta pedagógica se realizó la evaluación post-test a los niños, bajo las mismas condiciones en las cuales se efectuó el pretest.

\section{Propuesta pedagógica: "Pequeños exploradores de la ciencia"}

Esta propuesta tuvo como objetivo promover el pensamiento científico en niños del nivel preescolar en una institución educativa departamental de la zona bananera del departamento del Magdalena, como parte del currículo de la educación preescolar para la enseñanza de las ciencias. Se construyó a partir de situaciones de enseñanza contextualizadas, estrategias divertidas y significativas (adecuadas a las necesidades del niño) que son planteadas como problemas, desafíos o preguntas para permitir al infante buscar respuestas y elaborar explicaciones. La propuesta se estructuró a partir de 12 actividades y se desarrolló en dos meses, dependiendo los procesos de los niños; para ello, se estimó una temporalidad de 22 semanas con una intensidad horaria de 60 minutos en una jornada académica de 8:00 a 11:30 a.m. Las diferentes actividades contempladas en la propuesta se desarrollaron no solo en los espacios del aula de preescolar, sino que se requirió la adecuación y el uso de otros espacios físicos (dentro y fuera de la institución) con el propósito de acercar a los infantes a la exploración y reconocimiento de su entorno que hace posible la observación, formulación de preguntas, hipótesis y realización de inferencias; todos procesos cognitivos ligados al pensamiento científico.

Fue necesario describir y caracterizar las denominadas habilidades científicas o cognitivas, también Ilamadas habilidades del proceso científico, habilidades básicas para investigar o habilidades presentes en la mayoría de situaciones que conllevan a la formación científica como finalidad educativa. Puche-Navarro (2005b) propone los siguientes procesos cognitivos como herramientas o habilidades que están en la base del pensamiento científico: la clasificación, la planeación, la formulación de hipótesis, la inferencia y la experimentación.

La clasificación es una operación fundamental del pensamiento científico. Esta habilidad permite a los niños establecer diferentes tipos de criterios de diferencias y semejanzas (Puche-Navarro, 2005a), a partir de los cuales pueden organizar o sistematizar el conocimiento y la información que tienen de los fenómenos u objetos de su entorno. En otras palabras, la clasificación es la base para pensar científicamente, en tanto que los niños cuando sistematizan la información; en primer lugar, realizan sus propias colecciones o grupos y después establecen cualidades comunes de igualdad y semejanza entre los miembros de un grupo y, según esto, establecen clases o categorías de esos objetos (Puche-Navarro, 2005b).

La experimentación se refiere al proceso por el cual la teoría se vincula a la realidad por medio de la práctica; es probar y examinar una cosa o situación tantas veces que se permita descubrir, comprobar o demostrar hipótesis previas. Es así como los resultados de las comprobaciones van a determinar la decisión de mantener o rechazar dichas hipótesis. La experimentación es una herramienta por excelencia, la cual está estructurada por procedimientos y actividades que permiten obtener respuestas a los problemas planteados (Puche-Navarro y Ordóñez, 2003). 
La formulación de hipótesis, muy ligada a la experimentación, está relacionada con el hecho de formular preguntas, conjeturas, dudas y alternativas sobre la realidad considerada como hipótesis (Puche-Navarro, 2005a). Cuando el niño formula una hipótesis trata de representar su entendimiento ante las situaciones e ideas que enfrenta en la resolución de un problema que enfrenta. Según Karmiloff-Smith (1992), se trata de representaciones mentales de la manera como se entienden las situaciones y las ideas que tienen las personas para poder resolverlas.

En cuanto a la inferencia, desde antes de los dos años de edad los niños se muestran capaces de extraer inferencias de eventos que ocurren en situaciones cotidianas, en las cuales hacen uso de objetos que le plantean un problema a resolver (Cerchiaro, 2014). De acuerdo con Puche-Navarro (2005a), al inferir el niño hace conjeturas, establece relaciones entre la evidencia e hipótesis y busca soluciones hipotéticas a la problemática; de manera que los niños pueden extraer conclusiones sobre algo que no es directamente observable, a partir de información que ya se ha obtenido. La inferencia conduce a los niños a ir más allá de la información dada sobre un fenómeno que ellos desean comprender; de esta manera amplían su conocimiento, ejercitan sus habilidades de indagación, hacen búsquedas sistemáticas, analizan la información y organizan sus ideas en la generación de soluciones y de explicaciones en distintos contextos.

La planificación alude a la capacidad del niño para establecer un orden y una secuencia en las acciones de anticipación y previsión para conseguir un fin, así como prever y anticipar estrategias a estas acciones, permitiendo la posibilidad de repensar una situación propuesta. Para Puche-Navarro et al. "la Planificación es el proceso de generación de representaciones (posiblemente parciales) del comportamiento futuro antes de utilizar estas representaciones o planes para restringir o controlar dicho comportamiento" (2001, p. 34). En los niños la planificación se evidencia con acciones para conseguir un fin y con la corrección de las estrategias para manejar un problema en su totalidad (Guevara y Puche-Navarro, 2015).

Se puede afirmar que el pensamiento científico desde el preescolar, como lo menciona Vázquez (2014), ofrece múltiples ventajas en la vida diaria y en la formación del niño como persona, dado que el razonamiento y la lógica le ayudarán a responder ante los problemas, adaptarse a nuevas situaciones y solucionar retos. Puche-Navarro (2005a y 2005b) cuestiona la concepción que se tiene en la búsqueda de las condiciones que evoquen el funcionamiento de las capacidades tempranas de los niños como requisito importante en el trabajo del docente. Para poner al descubierto las capacidades cognitivas de los niños, esta autora propone lo siguiente: "Lo ideal es observar al niño utilizar las potencialidades descubiertas y adecuar sus propias situaciones, en este nivel observar al niño en su hábitat natural es absolutamente fundamental" (Puche-Navarro, 2005a, p. 44)

El niño pequeño procede de modo similar al científico que construye teorías acerca del mundo, formula y prueba hipótesis frente a la realidad que observa y trata de comprender (Karmiloff-Smith e Inhelder, 1974). Este planteamiento sirve de fundamento a la propuesta pedagógica "Pequeños exploradores de la ciencia", que se estructura mediante un plan de aula en el cual las ciencias naturales y el desarrollo del pensamiento científico del niño ocupan un lugar importante a través del estudio de la naturaleza, de los seres que la habitan, de las relaciones entre ellos, así como de la comprensión de los fenómenos que ocurren en ella, sus causas y sus consecuencias. Los fundamentos conceptuales que contempla la propuesta se relacionan en la figura 1.

En la tabla 2 se presentan los componentes de la propuesta pedagógica "Pequeños exploradores de la ciencia" y en la tabla 3 las experiencias didácticas desarrolladas. 
Tabla 2. Componentes de la propuesta pedagógica "Pequeños exploradores de la ciencia"

\section{Experiencia $\mathrm{n}^{\circ}$}

\section{Nombre de la experiencia}

Objetivo Que va a aprender el estudiante a través de la experiencia.

\begin{tabular}{|c|c|c|c|c|c|}
\hline $\begin{array}{l}\text { Derechos básicos de } \\
\quad \text { aprendizaje }\end{array}$ & \multicolumn{5}{|c|}{$\begin{array}{l}\text { Conjunto de aprendizajes estructurantes que construyen las niñas y los niños a través de las } \\
\text { interacciones que establecen con el mundo, con los otros y consigo mismos, por medio de } \\
\text { experiencias y ambientes pedagógicos en los que está presente el juego, las expresiones artísticas, la } \\
\text { exploración del medio y la literatura. }\end{array}$} \\
\hline $\begin{array}{l}\text { Evidencias de } \\
\text { aprendizaje }\end{array}$ & \multicolumn{5}{|c|}{$\begin{array}{l}\text { Muestran posibles manifestaciones del aprendizaje y un ejemplo que ilustra situaciones en las que } \\
\text { estos se hacen visibles. }\end{array}$} \\
\hline Materiales & \multicolumn{5}{|c|}{$\begin{array}{l}\text { Los implementos utilizados están relacionados con su contexto y se describen de forma particular } \\
\text { en cada una de las actividades. }\end{array}$} \\
\hline \multirow[b]{2}{*}{$\begin{array}{l}\text { Habilidad cognitiva/ } \\
\text { procedimiento }\end{array}$} & $\begin{array}{l}\text { Formular } \\
\text { preguntas }\end{array}$ & $\begin{array}{l}\text { Plantear } \\
\text { hipótesis }\end{array}$ & $\begin{array}{l}\text { Buscar } \\
\text { evidencias, } \\
\text { analizar y } \\
\text { trabajar en } \\
\text { equipo }\end{array}$ & Comunicar & Compromiso \\
\hline & $\begin{array}{l}\text { Observar } \\
\text { Este es el proceso } \\
\text { de mirar de } \\
\text { cerca, notar } \\
\text { desde diferentes } \\
\text { puntos de vista y } \\
\text { silenciosamente } \\
\text { observar y esperar } \\
\text { sin mucho } \\
\text { "hacer". } \\
\text { Comparar } \\
\text { Hacer } \\
\text { comparaciones } \\
\text { exhorta a los } \\
\text { niños a ir más allá } \\
\text { de decir lo que } \\
\text { notaron acerca de } \\
\text { algo y comenzar } \\
\text { a expresar } \\
\text { relaciones entre } \\
\text { las cosas. } \\
\text { Se establecen } \\
\text { las preguntas } \\
\text { generadoras, } \\
\text { reflexivas y } \\
\text { orientadoras. }\end{array}$ & $\begin{array}{l}\text { Ordenar, } \\
\text { Organizar y } \\
\text { predecir. } \\
\text { Es el proceso } \\
\text { de agrupar } \\
\text { cosas por rasgos } \\
\text { reconocibles. } \\
\text { Los niños } \\
\text { emparejan, } \\
\text { agrupan y } \\
\text { organizan } \\
\text { materiales de } \\
\text { muchas maneras } \\
\text { diferentes. } \\
\\
\text { Es el proceso de } \\
\text { cuestionamiento } \\
\text { y especulación, } \\
\text { basado en el } \\
\text { conocimiento } \\
\text { previo adquirido } \\
\text { en los tres } \\
\text { primeros pasos. } \\
\text { Los niños mejoran } \\
\text { en la predicción } \\
\text { a través de la } \\
\text { experiencia. }\end{array}$ & $\begin{array}{l}\text { Se mencionan los } \\
\text { materiales que se } \\
\text { van a utilizar. } \\
\text { Se indican } \\
\text { las normas } \\
\text { de seguridad } \\
\text { para realizar el } \\
\text { experimento. } \\
\text { Se explican los } \\
\text { pasos a seguir } \\
\text { durante el } \\
\text { desarrollo del } \\
\text { experimento. }\end{array}$ & $\begin{array}{l}\text { Evaluar } \\
\text { Los niños } \\
\text { comunican los } \\
\text { resultados de sus } \\
\text { experimentos con } \\
\text { otros, tomando } \\
\text { su experiencia } \\
\text { concreta, } \\
\text { verbalizándola y } \\
\text { representando la } \\
\text { información de } \\
\text { forma abstracta } \\
\text { con gráficos, } \\
\text { dibujos, gráficos y } \\
\text { libros de campo. } \\
\text { Aplicar } \\
\text { Este paso implica } \\
\text { la aplicación de } \\
\text { los conocimientos } \\
\text { adquiridos en } \\
\text { el experimento } \\
\text { a un campo } \\
\text { más amplio de } \\
\text { la experiencia, } \\
\text { alentando a los } \\
\text { niños a extender } \\
\text { el alcance de sus } \\
\text { experimentos, } \\
\text { probarlos de } \\
\text { nuevo con nuevos } \\
\text { materiales y ver si } \\
\text { su comprensión } \\
\text { es coherente. }\end{array}$ & $\begin{array}{l}\text { Este es el } \\
\text { momento } \\
\text { en el que se } \\
\text { proporciona } \\
\text { al niño la } \\
\text { posibilidad de } \\
\text { ampliar y afianzar } \\
\text { lo aprendido } \\
\text { mediante las } \\
\text { actividades } \\
\text { extracurriculares. }\end{array}$ \\
\hline
\end{tabular}

Fuente: elaboración propia. 


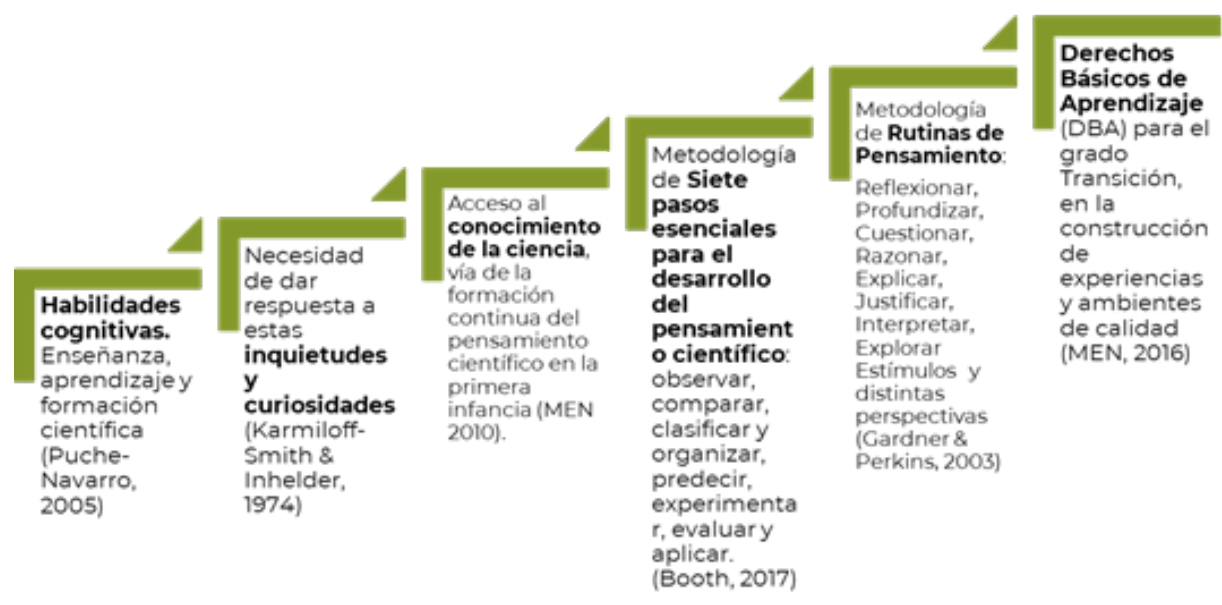

Figura 1. Fundamentos conceptuales de la propuesta.

Fuente: elaboración propia.

Tabla 3. Experiencias didácticas para

"Pequeños exploradores de la ciencia"

\section{Experiencias didácticas desarrolladas}

\begin{tabular}{ll}
\hline 1. & Descubriendo colores \\
\hline 2. & Mi amiga la planta \\
\hline 3. & Cambio el color de las flores \\
\hline 4. & Juguemos a las adivinanzas con los vegetales \\
\hline 5. $\quad$ Ciclo de vida de los animales \\
\hline 6. $\quad$ ¡Vamos hacer un delicioso pincho con productos de \\
\hline 7. ¿Blandon o duro? \\
\hline 8. ¿Qué le pasó al huevo? \\
\hline 9. Descubrimiento de mi amiga la gota \\
\hline 10. Conozcamos los estados del agua \\
\hline 11. Juguemos con la luz y la sombra \\
\hline 12. ¿Flotas o te hunde? \\
\hline 13. ¿Qué cae más rápido? La ley de gravedad
\end{tabular}

Fuente: elaboración propia.

\section{Resultados}

El análisis descriptivo de los resultados cuantitativos, tanto del pretest como del postest, está sujeto a la metodología de conglomerados jerárquicos (clúster). Esta es definida por Pérez (2008) como una técnica multivariante descriptiva y no inferencial, cuyo fin es agrupar o clasificar elementos mediante la formación de grupos o conglomerados (clúster). Esta dinámica posibilita la creación de subgrupos entre datos que comparten características similares, los cuales se siguen aglomerando en grupos cada vez más grandes; de ahí el nombre de jerárquico (Pérez, 2008). Para realizar este análisis se utilizaron las puntuaciones típicas de cada niño obtenidas en cada variable evaluada. El criterio para determinar la etapa en la que se configuran los grupos es que cada niño analizado pertenezca, según sus resultados, a un grupo de niños con puntuaciones semejantes.

Esta técnica permite comparar los cambios ocurridos en una de las variables del estudio, a través de las mediciones realizadas de las habilidades cognitivas de los niños de nivel preescolar mediante las escalas Merrill-Palmer (MP-R), las cuales fueron: cognición, motricidad fina, lenguaje receptivo, memoria, velocidad de procesamiento y coordinación visomotora.

En el análisis de los resultados, en primera instancia, se aplicó la prueba Wilcoxon para comparar los resultados de cada variable tanto en pretest como en postest. En la tabla 4 se muestran los valores $Z$ y el nivel de significancia arrojados por esta prueba. 
Tabla 4. Valores Z y nivel de significancia de la prueba de Wilcoxon

\begin{tabular}{|c|c|c|c|c|c|c|c|}
\hline \multirow[t]{2}{*}{ Z } & $\begin{array}{l}\text { Índice } \\
\text { Global }\end{array}$ & Cognición & $\begin{array}{l}\text { Motricidad } \\
\text { fina }\end{array}$ & $\begin{array}{l}\text { Lenguaje } \\
\text { receptivo }\end{array}$ & Memoria & $\begin{array}{l}\text { Velocidad de } \\
\text { procesamiento }\end{array}$ & $\begin{array}{c}\text { Coord. } \\
\text { visomotora }\end{array}$ \\
\hline & $-3,296$ & $-2,972$ & $-1,992$ & $-2,922$ & $-2,417$ &,- 384 & $-2,489$ \\
\hline $\begin{array}{l}\text { Sig. asintótica } \\
\text { (bilateral) }\end{array}$ &, $001^{*}$ &, $003 *$ &, $046^{*}$ &, $003 *$ &, $016^{*}$ & ,701 &, $013 *$ \\
\hline
\end{tabular}

Fuente: elaboración propia.

La comparación de resultados de la muestra de estudio mediante Wilcoxon demuestra que existen diferencias significativas entre el pretest y postest en cuanto al índice global ( $\mathrm{P}=0,001)$, la cual es una medida general del desarrollo del niño que abarca la cognición, el lenguaje y la motricidad fina. También, existen diferencias significativas entre las variables de cognición $(\mathrm{P}<0,05)$, motricidad fina $(P<0,05)$, lenguaje receptivo $(P<0,05)$, memoria $(\mathrm{P}<0,05)$ y coordinación visomotora $(\mathrm{P}<0,05)$; esto indica variaciones entre los resultados del pretest $y$ postest en las variables mencionadas. Por otro lado, la velocidad de procesamiento en la muestra del estudio no presenta diferencias significativas en las dos mediciones realizadas.

En términos generales, se evidencian cambios en el número y tamaño de los grupos conformados mediante la metodología de análisis de clústeres en la muestra de estudio, configurándose así tres grupos en el pretest y dos grupos en el postest. En el pretest existen dos grupos que contienen la mayoría de los niños de la muestra, y uno más pequeño en el cual se agrupan solo dos niños. En el postest se conforma un gran grupo con 10 niños y uno más reducido integrado por cuatro niños. Lo que esto indica es que los resultados de los niños de cada grupo son más parecidos en el prestest, mientras que en postest tienden a ser más variables. Para comprender estos resultados es necesario obtener las puntuaciones de los conglomerados y compararlos entre sí para determinar los cambios producidos antes y después de la intervención.

Las puntuaciones típicas de los niños de cada grupo fueron promediadas para obtener perfiles grupales que permitieran detallar las diferencias de resultados entre las agrupaciones conformadas. Ya que se emplearon las puntuaciones típicas para construir los perfiles grupales, se mantuvo entonces la media $(M=100)$ y la desviación típica $(D t=15)$ planteadas en las escalas de desarrollo Merrill-Palmer-R (MP-R). A continuación, se presentan las gráficas que detallan los perfiles grupales conformados en el Pretest y Postest.

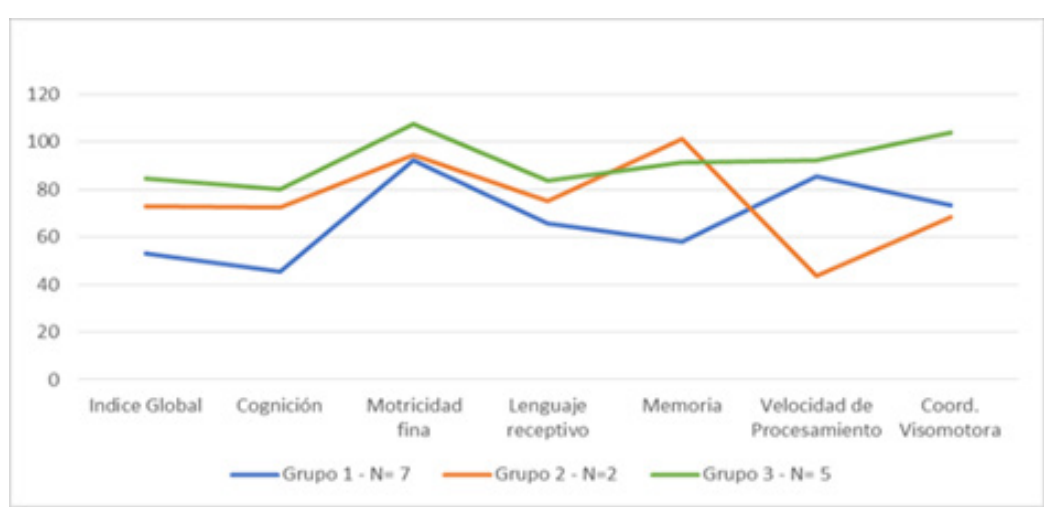

Figura 2. Clústeres que muestran perfiles grupales en el pretest.

Fuente: elaboración propia. 


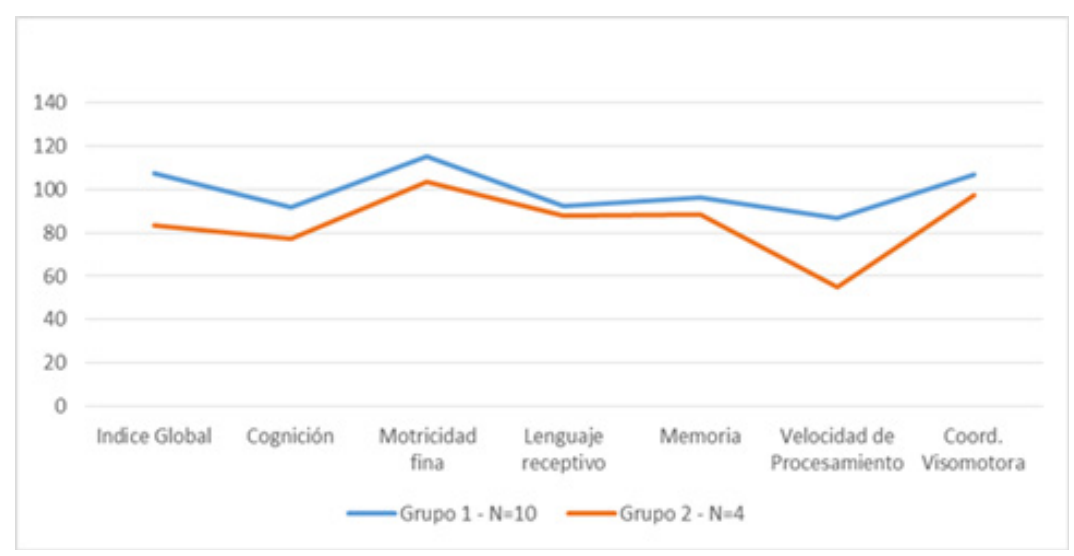

Figura 3. Clústeres que muestran perfiles grupales en el post-test.

Fuente: elaboración propia.

Como se observa en la figura 2, en el pretest el grupo 1, donde se encuentra la mayoría de niños $(\mathrm{N}=7 ; 50 \%)$, hay en todas las variables evaluadas puntuaciones por debajo de la media. En esta misma medición, el grupo $3(\mathrm{~N}=5 ; 35,7 \%)$ obtuvo los mejores resultados de toda la muestra (a excepción de la variable memoria), encontrándose la mayoría de sus puntuaciones cercanas a la media y sobrepasando esta medida en motricidad fina y velocidad de procesamiento. Los niños del grupo 2 ( $N=2 ; 14,3 \%$ ) también alcanzaron, en la mayoría de las variables evaluadas, puntuaciones cercanas a la media, aunque más bajas que las obtenidas por el grupo 3. Estos últimos niños obtuvieron las mayores puntuaciones de toda la muestra en los ítems que evaluaban memoria; no obstante, también tuvieron el más bajo desempeño en velocidad de procesamiento.

En general, en los resultados del pretest conseguidos por todos los niños de la muestra se registran puntuaciones por debajo de la media en la mayoría de las variables consideradas en este estudio. Las puntuaciones más bajas se encuentran en el área de cognición y las más altas fueron alcanzadas en motricidad fina.

Los resultados del postest indican una mejora considerable en las puntuaciones de los niños en relación con el pretest. Los dos grupos muestran un desempeño que los ubica muy cerca de la media estandarizada. El grupo $1(\mathrm{~N}=10)$, el cual recoge al $71,4 \%$ de los niños de la muestra de estudio es el que alcanza las mejores puntuaciones. En este caso, los puntajes del índice global sobrepasan la media, al igual que los de motricidad fina y coordinación visomotora. El grupo $2(\mathrm{~N}=4)$, que representa el 29,6\% de la muestra, también obtuvo en el postest resultados cercanos a la media en la mayoría de las variables evaluadas; a excepción de velocidad de procesamiento y cognición, donde se evidencian los resultados más bajos.

Aunque los resultados de los niños participantes en los dos grupos del clúster, durante el postest, indican que los desempeños más bajos fueron obtenidos en los ítems que miden la cognición y la velocidad de procesamiento, tal y como se evidencia en el pretest, se atestigua una mejora considerable en esta última medición, respaldada en un aumento en las puntuaciones alcanzadas en estas dos variables mencionadas. Esta mejora se puede apreciar con mayor claridad en el área de cognición, la cual demostró cambios significativos entre pretest y postest a partir del análisis arrojado por la prueba estadística Wilcoxon. No obstante, el aumento en los puntajes alcanzados por los niños durante el postest (en velocidad de procesamiento) no fueron suficientes para que se determinen cambios estadísticamente significativos mediante Wilcoxon.

Se encuentran diferencias que acreditan cambios en los resultados obtenidos por los niños en el pretest y postest. Uno de estos cambios es la desaparición de uno de los tres grupos conformado en el pretest para dar lugar a la configuración de solo dos grupos en el postest (figura 4). El grupo 1, constituido en su mayoría por los niños que se encontraban en 
los grupos 1 y 2 del pretest; y el grupo 2, que duplica en número al grupo 3 del pretest.

Vale la pena recordar que cada grupo conformado da cuenta de un perfil con respecto a las habilidades cognitivas evaluadas, que guarda relación con el nivel de desarrollo alcanzado por los niños. Los resultados del postest indican que hubo un cambio en estos perfiles. Esto podría significar, de acuerdo con inferencias lógicas, que los niños no solo mejoraron su rendimiento en las distintas pruebas realizadas, sino que la intervención en la que participaron disminuyó las diferencias entre ellos en relación con las habilidades cognitivas evaluadas. Sin embargo, ante la falta de un grupo control que permitiera comparar la manifestación de estos cambios no es posible confirmar una relación causal entre las variables estudiadas que justifiquen los cambios operados.

Se recomienda en futuras investigaciones incorporar en el diseño un grupo control que permita hacer más evidente el papel de la variable independiente sobre la variable dependiente. Para que, de esta manera, pueda darse mayor validez al cambio en las habilidades cognitivas de los niños como efecto de su participación en la propuesta pedagógica desarrollada.

Después de revisar los resultados cuantitativos de la evaluación pretest y postest, se hace necesario presentar los datos que a nivel cualitativo se desprenden de la observación realizada durante la implementación de la propuesta pedagógica. El análisis que se deriva de estas observaciones destaca los siguientes aspectos: la actitud y respuesta de los niños y niñas a las experiencias propuestas, las habilidades científicas trabajadas y las fortalezas y debilidades de la propuesta.

\section{Actitudes e implicación del alumnado}

En cada una de las experiencias los niños, en su mayoría, se mostraron muy interesados y participativos; se comprometieron con la experiencia, realizándola desde principio a fin. En general, mostraron una actitud positiva, ya que las diferentes experiencias se ajustaron a su nivel cognitivo e intereses propios de su edad cronológica, lo que les permitió involucrarse favorablemente. Cabe resaltar que mediante las estrategias didácticas basadas en el juego se les brindó la oportunidad de realizar experimentos con materiales y elementos cercanos a ellos, ya que estaban relacionados con su vida cotidiana. Esta actitud positiva de los niños y niñas se hizo evidente en su disposición de participar de manera voluntaria, en el compromiso con la tarea asociado a la permanencia en el marco de las acciones propuestas $y$, finalmente, en el seguimiento de instrucciones por parte de cada uno de ellos; todo ello garantizó la completa aplicación de cada una de las experiencias de la propuesta pedagógica.

\section{Habilidades científicas trabajadas}

Las experiencias realizadas con los niños, en general, propiciaban el despliegue de habilidades científicas como la clasificación, la experimentación, la inferencia, la planeación y la formulación de hipótesis atendiendo a las demandas cognitivas planteadas. Cabe aclarar que de estas habilidades unas eran requeridas en mayor medida que otras, dependiendo de la experiencia propuesta.

\section{Fortalezas y debilidades de la propuesta pedagógica}

En cuanto a los aspectos que funcionaron adecuadamente en el marco de la propuesta se plantean en primer lugar los espacios seleccionados para el desarrollo de las experiencias, los cuales permitieron mantener un adecuado nivel de curiosidad en los niños y la indagación necesarios para llevar a cabo todas las experiencias de manipulación y experimentación con materiales de su medio. Los terrenos que rodean la escuela se convirtieron en lugares de exploración, en los que se disponía de diferentes muestras (plantas, animales, rocas) para ser observadas y analizadas.

En segundo lugar, Ilama la atención el asombro y la disposición que la mayoría de los niños y niñas mostró al participar en cada una de las experiencias propuestas; saber que cada una permitía explorar lúdicamente sus habilidades científicas fue enriquecedor. La combinación de colores, la formación de las nubes, la germinación de una semilla, la creación de material viscoso, el cambio de color de los pétalos de las flores, la sesión de 
adivinanzas sobre las frutas, aprovechamiento de su contexto para que los niños y niñas explicaran el significado que tiene experimentar con el aire y con el agua, entre otras, fueron experiencias que pusieron de manifiesto emociones, motivación y dinamismo por parte de los niños. Cada cual posibilitó el despliegue cognitivo a partir de la estructuración de nuevos conocimientos asociados con la ciencia, puesto que los niños y niñas hacían conjeturas acerca de lo observado, elaboraban predicciones en torno a lo que podría pasar, mientras cuidaban su trabajo teniendo en cuenta los detalles para que sucediera lo que se esperaba o lo que ellos esperaban; algunos estuvieron muy cercanos a la realidad, se apoyaban entre pares, incluso se complementaban en las ideas. Algunos escuchaban el aporte de sus compañeros y, en particular, generaban explicaciones puntuales, por ejemplo, explicaciones acerca de la formación de las nubes.

Como aspectos a mejorar en futuras experiencias pedagógicas de esta naturaleza la observación del proceso mostró que los niños están poco familiarizados con este tipo de experiencias, por lo que en ocasiones su comportamiento fue inadecuado (por ejemplo, todos querían hacer el experimento, entre ellos se peleaban las cosas, no permitían que todos observaran). Se hace necesario, entonces, incorporar este tipo de experiencias al currículo y hacer de ellas una práctica cotidiana y sistemática, en el marco de normas de conducta claras, las cuales crean un clima apropiado y agradable para trabajar los experimentos. Otra tarea inaplazable es la adecuación en el aula de un rincón con múltiples materiales para despertar en ellos la capacidad de descubrir y experimentar por sí solos, con la guía apropiada del maestro, como se propuso en el marco de esta propuesta.

Ligado a lo anterior, el tiempo establecido para el desarrollo de la propuesta pedagógica es un aspecto fundamental a tener en cuenta. Atendiendo a factores externos (como protestas sindicales y cambios en el calendario académico) fue necesario priorizar las experiencias y de un total de 16 experiencias se decidió aplicar solo 12, optimizando el tiempo lo máximo posible. La inasistencia de algunos de los niños participantes es otro aspecto que se estima debe ser mejorado.
Partiendo de las anteriores descripciones, se puede colegir que la aplicación de una estructura metodológica basada en aportes de autores como Piaget (1999), Puche-Navarro (2005a), Booth (2017) y los lineamientos del Ministerio de Educación Nacional $(2004,2014)$ posibilitó la creación de condiciones que favorecen el pensamiento científico en los niños al potenciar habilidades como la formulación de preguntas, el planteamiento de hipótesis o conjeturas acerca de lo que observan, la experimentación, la búsqueda y clasificación de evidencias, entre otras.

Asimismo, puede afirmarse que la presente propuesta pedagógica propició la formulación y desarrollo de experiencias ajustadas a los niños de preescolar. En esta se privilegió la utilización del dibujo como un medio para acceder a la manera cómo piensan, expresan lo que entienden y sienten acerca del mundo en que viven.

Se demuestra el valor pedagógico de la estrategia didáctica basada en el modelo de Booth (2017), en tanto que los pasos aplicados en el desarrollo de las experiencias científicas con los niños les permitió poner en juego habilidades científicas a partir de la observación de fenómenos o eventos que ocurren en el propio contexto. No obstante, en la aplicación de este modelo es necesario tener en cuenta algunos obstáculos que se presentaron asociados a la escasa familiarización de los niños con este tipo de actividades que los Ilevaban a pensar y a comunicar sus ideas. Se requiere, entonces, continuar proponiéndoles situaciones en las que ellos puedan actuar, manipular y experimentar al tiempo que se aprovecha su curiosidad, lo cual garantizará un nuevo aprendizaje y la construcción de un conocimiento significativo.

\section{Conclusiones}

La presente investigación tuvo como objetivo general promover el desarrollo del pensamiento científico en niños de nivel preescolar mediante la propuesta pedagógica "Pequeños exploradores de la ciencia", orientada a la enseñanza de las ciencias en una institución educativa departamental de la zona bananera, departamento del Magdalena. Las conclusiones que aquí se exponen dan cuenta de la caracterización de las condiciones (individuales, 
pedagógicas, curriculares, didácticas y contextuales) que a la luz de los resultados obtenidos favorecen el desarrollo del pensamiento científico en el nivel de preescolar.

\section{Condiciones individuales}

1. Los niños son capaces de hacer uso de habilidades propias del pensamiento científico.

2. Sobrepasan sus intuiciones, su sentido común y adaptan sus ideas a los hechos y acontecimientos partir de la observación de su entorno.

3. Los niños aprenden a partir de la experimentación, la observación y el seguimiento de patrones específicos, lo que les convierte en pequeños investigadores.

4. Sus experiencias reales derivadas de la interacción directa con los fenómenos o situaciones constituyen una fuente de información apropiada para construir sus propias justificaciones.

5. Los niños mostraron un cambio no solo en la manera como logran acercarse a un fenómeno o evento en términos cognitivos, sino también en su aproximación afectivo-motivacional.

Las condiciones individuales señaladas dan cuenta de "cómo aprenden los niños", quienes, según Piaget e Inhelder (2007), actúan como "pequeños científicos" que en su interacción con el entorno buscan interpretar el mundo, siguiendo su propia lógica y formas de conocer, de acuerdo con patrones predecibles del desarrollo conforme van alcanzando la madurez. Lo cual lleva a pensar en la necesidad de una educación que parta del desarrollo de los niños, es decir, desde sus capacidades reales y necesidades de formación (Cerchiaro, 2019).

\section{Condiciones pedagógicas y didácticas}

1. La enseñanza de las ciencias naturales en el nivel preescolar debe estar apoyada en estrategias didácticas que favorezcan el desarrollo de habilidades científicas (clasificación, experimentación, formulación de hipótesis, inferencias y planificación) a partir de experiencias científicas y lúdicas que fortalezcan la capacidad crítica, el razonamiento, la argumentación y la creatividad en el niño desde el preescolar.
2. Se valida el uso de actividades didácticas e incorporar un mayor número de situaciones u oportunidades para la adquisición de habilidades y destrezas relacionados con la ciencia.

3. Aplicar este modelo en actividades propias de otras áreas del currículo.

4. Se hizo evidente el que hacer del maestro como facilitador para el aprendizaje.

5. Se resalta la importancia de brindar en el nivel preescolar un mayor número de oportunidades o experiencias a través de las cuales los niños hagan un despliegue de sus habilidades científicas y la creación de espacios en donde la curiosidad, la exploración de objetos y la indagación desde su propio contexto resulte importante a la hora de fortalecer la capacidad crítica, el razonamiento, la argumentación y la creatividad en el niño desde el preescolar.

\section{Condiciones curriculares}

1. La pertinencia de la estructura curricular diseñada depende del fortalecimiento de procesos básicos.

2. Las bases en un currículo desde la experiencia cobran sentido en cuanto los niños interactúan, exploran e indagan obedeciendo a sus propios intereses y preguntas, en sus propias maneras de comunicarse, en su sensibilidad y desde su propio contexto.

3. El papel del docente es el de constructor permanente de situaciones y ambientes que dan vida a las experiencias que los niños y las niñas viven. Además, las reconstruye, enriquece y reorganiza para generar nuevas acciones que posibilitan nuevos aprendizajes (Dewey, citado en Ministerio de Educación Nacional, 2017).

4. El desarrollo del pensamiento científico va a depender del fortalecimiento de procesos básicos como: la observación, la clasificación, la seriación, el uso de los números, la medición, la inferencia, la predicción y la comunicación; los cuales son fundamentales para propiciar la promoción de aprendizajes significativos en el aula y para potenciar la adquisición de habilidades y actitudes científicas desde el preescolar, 
valorando la ciencia de manera positiva.

Finalmente, se puede destacar que la labor docente que favorece la investigación científica de los estudiantes es aquella que se apoya en la observación, la reflexión y la experimentación.

\section{Recomendaciones}

- Establecer con mayor regularidad experiencias enriquecedoras a través de estrategias lúdicas y significativas basadas en la experimentación, exploración e indagación y que vayan según los intereses de los niños, consecuentes para trabajar con los proyectos de aula.

- Incorporar al proceso de enseñanza-aprendizaje de las ciencias, estrategias y herramientas cognitivas basadas en experiencias pedagógicas para mediar el desarrollo del pensamiento científico, desde su propio contexto.

- Incluir en el aula un "Rincón de las ciencias" para favorecer la actitud de los niños frente a las ciencias y sus aprendizajes.

- Disponer de recursos y materiales didácticos reciclables (crear pequeñas colecciones).

- Uso de recursos digitales (como videos, internet, etc.) a través de los cuales los niños puedan acceder a información y tener contacto (virtual) con situaciones, lugares, materiales y experiencias que promuevan su pensamiento científico.

\section{Referencias}

Alcantud, F., Yurena, A. y Rico, D. (2015). Validez y fiabilidad del sistema de detección precoz de los trastornos del desarrollo: 3 a 36 meses. Revista Española de Discapacidad, 3(1), 107-121. https://doi.org/10.5569/2340-5104.03.01.06

Alonso, Y. (2014). Validación del sistema de detección precoz de trastornos del desarrollo (SDPTD). 3 a 12 meses. Universitat de València.

Arribas, D. y Pereña, J. (2011). CompeTEA. Tea Ediciones. http://web.teaediciones.com/COMPETEA.aspx

Ausubel, D., Novak, J. y Hanesian, H. (1983). Psicología educativa. Un punto de vista cognoscitivo. Trillas.
Bick, J. (2015). Experiencias negativas tempranas: ¿qué hemos aprendido con las últimas investigaciones sobre el cerebro? Revista Espacio para la Infancia, 43, 10-13.

Booth C., E. (2017). Scientific Thinking: Step by Step. Early Childhood Today. Virtual Magazine. https://www.scholastic.com/teachers/articles/ teaching-content/scientific-thinking-step-step/

Cerchiaro, E. (2014). Emergencia, patrones de cambio y trayectorias de desarrollo en niños entre 15 y 26 meses de edad (tesis de doctorado no publicada). Universidad del Valle.

Cerchiaro, E. (2019). La escuela infantil como espacio para el desarrollo. En E. Cerchiaro (comp.), Acciones educativas en la escuela para la primera infancia. Editorial Unimagdalena.

Cypel, S. (2013). Lo que ocurre en el cerebro de los niños muy pequeños durante el proceso de aprendizaje. Espacio para la Infancia, 39, 14-19.

De Zubiría, J. (2013). El maestro y los desafíos a la educación en el siglo XXI. Revista Redipe, 825, 12.

Guevara, M. y Puche-Navarro, R. (2015). The Emergence of Cognitive Short -Term Planning: Performance of Preschoolers in a Problem-Solving Task. Acta Colombiana de Psicología, 18(2), 13-27.

Harlen, W. (1998). Enseñanza y aprendizaje de las ciencias. Ediciones Morata.

Hernández, R., Fernández, C. y Baptista, P. (2006). Metodología de la investigación. McGraw Hill.

Jácome-Vera, C. (2016). El proceso de rehabilitación neuropsicológica de un niño con retraso cognitivo y de lenguaje oral, en relación al desarrollo evolutivo según Piaget, con la aplicación de las escalas de desarrollo MerrillPalmer- $R$ (tesis de Maestría en Educación Especial). Universidad Tecnológica Equinoccial.

Karmiloff-Smith, A. (1994). Más allá de la modularidad. Alianza.

Rico, D. (2013). Construcción y validación de un sistema de detección precoz de los trastornos del desarrollo (SDPTD): 18 a 36 meses. Universitat de València.

Roid, G. H. y Sampers, J. L. (2004). Escalas de desarrollo Merrill-Palmer (MP-R). Tea Ediciones. 
Samper, R. H. (2013). Los métodos mixtos. Editorial Mcgraw Hill.

Torrado, M., Reyes, M. y Durán, E. (2006). Bases para la formulación de un plan nacional para el desarrollo de la primera infancia. Revista Infancia Adolescencia y Familia, 1(1), 15-38.

Vygotski, L. S. (1979). El desarrollo de los procesos psicológicos superiores. Crítica.

Wilcoxon, F. (1945). Individual comparisons by ranking methods. Biometrics Bulletin. https:// doi.org/10.2307/3001968

Winerman, L. (2017). 4 questions for Alison Gopnik. The Developmental Psychologist explains why we can't raise "monoculture" kids. Monitor on Psychology, 48(3), 33-34.

Yáñez, L. (2013). Aprendiendo desde el nacimiento. Espacio para la Infancia, 39, 5-6.

Karmiloff-Smith, A. e Inhelder, B. (1974). If you want to get ahead, get a theory. Cognition, 3, 195-212. https://doi.org/10.1016/0010-0277(74)90008-0

Kerlinger, F. y Lee, H. (2002). Investigación del comportamiento. Método de investigación en ciencias sociales. McGraw-Hill.

Ministerio de Educación Nacional de la República de Colombia (2004). Estándares básicos de competencias en Ciencias Naturales y Ciencias Sociales. Ministerio de Educación Nacional de la República de Colombia.

Ministerio de Educación Nacional de la República de Colombia (2014). La exploración del medio en la educación inicial (documento $n .^{\circ}$ 24). Ministerio de Educación Nacional de la República de Colombia.

Ministerio de Educación Nacional de la República de Colombia (2017). Bases curriculares para la educación inicial y preescolar. Ministerio de Educación Nacional de la República de Colombia.
Monsalve, A., Cubillos, N., Peña, M. y Peñaranda, A. (2015). Características de la intervención ocupacional en un caso de trastorno del espectro autista. Revista Ocupación Humana, 15(1), 5-18. https://doi.org/10.25214/25907816.38

Orozco, B. C., Puche, R., Orozco, M. y Correa, M. (2009). Desarrollo infantil y competencias en la Primera Infancia. Ministerio de Educación Nacional de la República de Colombia.

Pérez, C. (2008). Técnicas de análisis multivariante de datos. Aplicaciones con SPSS. Pearson Prentice Hall.

Perkins, D. T. (1998). Un aula para pensar: Aprender y enseñar en una cultura del pensamiento. Aique.

Piaget, J. (1969). El desarrollo de la inteligencia en el niño. Aguilar.

Piaget, J. (1999). La psicología de la inteligencia. Crítica.

Piaget, J. e Inhelder, B. (2007). Psicología del niño. Morata

Puche-Navarro, R. (2005a). Formación de herramientas científicas en el niño pequeño. Artes Gráficas del Valle.

Puche-Navarro, R. (2005b). Los comienzos de la experimentación y la racionalidad mejorante en el niño. En, Formación de herramientas científicas en el niño pequeño (pp. 13-44). Artes Gráficas del Valle.

Puche-Navarro, R. y Ordóñez, O. (2003). Pensar, experimentar y volver a pensar: un estudio sobre el niño que experimenta con catapultas. En R. Puche-Navarro, El niño que piensa y vuelve a pensar (pp. 88-177). Artes Gráficas del Valle. Puche-Navarro, R., Colinvaux, D. y Divar, C. (2001). El niño que piensa. El niño: científico, lector, escritor y matemático. Universidad del valle. 